\title{
The Use of High Resolution Computerized Tomography (HRCT) of the Chest in Evaluating the Effect of Tobramycin Solution for Inhalation in Cystic Fibrosis Lung Disease
}

\author{
Samya Z. Nasr, MD, ${ }^{1 *}$ Ermelinda Sakmar, MA, RN, ${ }^{1}$ Emmanuel Christodoulou, $\mathrm{PhD},{ }^{2}$ \\ Boris P. Eckhardt, MD, ${ }^{3}$ Daniel S. Streetman, PharmD, MS, ${ }^{4}$ and Peter J. Strouse, $\mathrm{MD}^{3}$
}

Summary. Objectives: To compare the usefulness of HRCT of the chest versus spirometric measures (PFTs) in evaluating the effect of tobramycin solution for inhalation (TSI) in cystic fibrosis (CF). Methods: Thirty-two CF patients with mostly mild lung disease age $\geq 6$ years, were enrolled in a double-blind, placebo-controlled pilot study. Patients were chronically colonized with Pseudomonas aeruginosa for at least 6 months prior to and at enrollment. If patients were on TSI, they were taken off for at least 3 months prior to enrollment. Duration was 6 months; 31 subjects completed the study. HRCT and PFTs were evaluated at baseline, after 28 days of treatment and at the end of the study. Study medication was administered as $5 \mathrm{ml}$ nebulized treatment twice a day for 28 days followed by 28 days off (one cycle). Study consisted of three cycles. Two radiologists scored all films using a validated system. A total HRCT score consists of the sum of subscores: linear opacities, hyperinflation, nodular opacities, peribronchial thickening, mucous plugging, and bronchiectasis; each subscore could range from 0 to 80 , with potential total scores varying from 0 to 480 . The percent of the maximum possible HRCT score was then calculated and used for all comparisons. Results: Using two tailed paired $t$-test, the percent maximum HRCT score decreased by $1.4 \pm 2.6 \%$ (mean \pm SD) $(P=0.049)$ and $0.3 \pm 2.8 \%$ $(P=0.63)$ for the TSI group and decreased by $0.1 \pm 1.5 \%(P=0.74)$ and increased by $0.6 \pm 1.8 \%$ $(P=0.23)$ for the placebo group between visits 1 and 2 , and visits 1 and 3 , respectively. The data were then analyzed using a mixed model utilizing changes in scores over the durations of the study for each group. The change of HRCT score for the TSI group was $-0.24 /$ day $(P=0.02)$ and -0.03 / day $(P=0.22)$, and for the control group the change was $-0.01(P=0.93)$ and $0.02(P=0.29)$ between visits 1 and 2 , and visits 1 and 3 respectively. $F F_{25-75} \%$ and $F E V_{1} \%$ changes were not statistically significant using both analyses. Conclusion: HRCT seems to be more sensitive in detecting treatment effect than PFT in CF patients with mild lung disease, especially following the first treatment period (visit 2). Total HRCT score showed some improvement at the end of the study, though not statistically significant. This is probably due to obtaining the HRCT an average of 30 days after completion of the TSI treatment, and selection of study population with mostly mild lung disease. This could indicate that the most significant improvement in the total HRCTscore in this patient population occurs after the first treatment period with TSI. Pediatr Pulmonol. 2010; 45:440-449. ๑ 2010 Wiley-Liss, Inc

Key words: cystic fibrosis; HRCT; $\mathrm{FEV}_{1} ; \mathrm{FEF}_{25-75 \%}$; TSI; Pseudomonas aeruginosa.

\footnotetext{
${ }^{1}$ Division of Pediatric Pulmonology, Department of Pediatrics, University of Michigan Health System, Ann Arbor, Michigan.

${ }^{2}$ Section of Physics/Engineering, University of Michigan Health System, Ann Arbor, Michigan.
}

${ }^{3}$ Department of Radiology, Section of Pediatric Radiology, University of Michigan Health System, Ann Arbor, Michigan.

${ }^{4}$ Metabolism, Interactions, and Genomics Group, Lexi-Comp, Inc., Hudson, Ohio.

Presented in part at the American Thoracic Society International Meeting, Toronto, Canada, May 20, 2008 and the NACF Meeting, Orlando, FL, October 23-26, 2008.

(C) 2010 Wiley-Liss, Inc.
Grant sponsor: Novartis.

*Correspondence to: Samya Z. Nasr, MD, Professor of Pediatrics, 1500 E. Medical Center Drive, Ann Arbor, MI 48109-5212.

E-mail: snasr@umich.edu

Received 4 December 2008; Revised 22 September 2009; Accepted 26 September 2009.

DOI $10.1002 /$ ppul.21188

Published online 2 April 2010 in Wiley InterScience

(www.interscience.wiley.com). 


\section{INTRODUCTION}

Infants with cystic fibrosis (CF) have normal lungs at birth. ${ }^{1}$ Early manifestation of lung disease starts shortly after birth. ${ }^{1}$ The predominant causes of early lung disease in $\mathrm{CF}$ are persistent infection and inflammation. ${ }^{2}$ These lead to chronic lung disease, progressive worsening in lung function, and increased morbidity and mortality. ${ }^{2}$ Median age of survival has been steadily increasing over the last few decades. Currently, the median survival is 37.4 years of age. ${ }^{3}$ Detection of early CF lung disease is essential for understanding the disease progression. ${ }^{4}$

It has been reported that in mild CF lung disease, the use of HRCT is more sensitive and superior to chest radiographs (CXR) in evaluating and monitoring lung structure. ${ }^{5,6}$ Pulmonary function tests (PFTs) are widely used to evaluate and monitor progression of lung disease in CF patients. However, they are insensitive in localizing early lung disease and are indirect measure of lung structure. ${ }^{7}$ Longitudinal studies using HRCT and spirometry in CF patients revealed significant changes in lung parenchyma, especially in early stages of the lung disease using HRCT. ${ }^{8-11}$ However, in these studies, patients had normal or stable spirometry findings. These findings support the notion that PFTs may not be an accurate measure for monitoring the progression of early lung disease. ${ }^{8-11}$

Tobramycin solution for inhalation (TSI) has been studied and shown effective in CF patients with moderate to severe lung disease with $\mathrm{FEV}_{1}$ between $25 \%$ and $75 \%$ predicted and chronically colonized with Pseudomonas aeruginosa. ${ }^{12}$ TSI was studied in CF patients with mild lung disease and chronic P. aeruginosa colonization. ${ }^{13}$ One study showed significant reduction in respiratory hospitalizations, antibiotic use, and improvement (not statistically significant) in percent predicted forced expiratory flow $\left.\left(\mathrm{FEF}_{25-75 \%}\right)\right)^{13}$ The purpose of this pilot study was to evaluate the effect of TSI on CF patients $\geq 6$ years with mostly mild lung disease and colonization with $P$. aeruginosa using HRCT and PFT as end points. We hypothesized that HRCT might be more effective than PFT in evaluating the effect of TSI on CF patients with mild-moderate lung disease. Previously, we reported results from the early part of the study. ${ }^{14}$ The HRCT

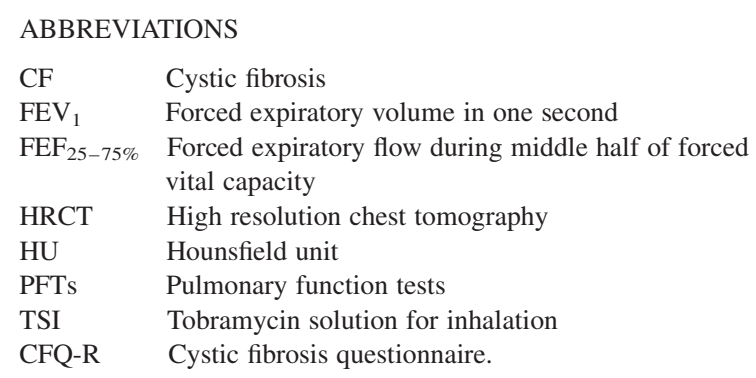

score used in the initial analysis was derived from Brody. ${ }^{11}$ That score consisted of eight component scores (bronchiectasis, peribronchial thickening, mucus plugging, atelectasis, nodular opacities, linear opacities, inhomogeneity, and hyperinflation). A modified score was calculated by dropping two discordant component scores (atelectasis and inhomogeneity). In this study we used the modified HRCT score in the analysis. In this article, we are reporting the results of the 6-month study assessing the effects of TSI versus placebo in this patient population.

\section{MATERIALS AND METHODS}

\section{Study Design}

This is a randomized, double-blind, placebo-controlled pilot study. The study procedures and measures were approved by the University of Michigan Institutional Review Board. Consent was obtained from patients and/or parents/guardians prior to enrollment in the study.

\section{Study Population}

Patients with CF 6 years of age and older with $\mathrm{FEV}_{1}$ $\geq 40 \%$ predicted, attending the University of Michigan CF Center, were considered eligible for the study. Thirty-two patients (12 males and 20 females) with a confirmed diagnosis of CF by sweat chloride test and/or genotype, and $P$. aeruginosa colonization in sputum obtained from deep pharyngeal culture, for at least 6 months of screening and at enrollment were recruited. Patients with first colonization with $P$. aeruginosa were not recruited. Exclusion criteria included known hypersensitivity to aminoglycosides or history of Burkholderia cepacia isolated from respiratory cultures. Patients had to be clinically stable prior to enrollment with no hospitalization or intravenous antibiotic use for pulmonary exacerbation within 2 months of enrollment. Also, no oral antibiotic use within 1 month of enrollment and no TSI use for at least 3 months prior to enrollment. All subjects were pancreatic insufficient.

\section{Study Procedures}

Enrollment duration was 18 months, from February 2002 to August 2003. Patients were evaluated at baseline, 28 days and at the end of the study. Eligible subjects were randomized to receive either $5 \mathrm{ml}$ of TSI or $5 \mathrm{ml}$ of placebo twice a day, via inhalation therapy for 28 days followed by 28 days off medication (one cycle). Study consisted of three cycles (6 month duration). TSI (Novartis, New Hanover, NJ) is a solution of $300 \mathrm{mg}$ tobramycin and $11.25 \mathrm{mg}$ sodium chloride in sterile water; the placebo was chosen for its similar taste to TSI and consisted of $1.25 \mathrm{mg}$ quinine sulfate in normal saline. Adherence to study treatments was determined by collecting and counting all used and unused medication vials that patients were asked 
to return. Routine CF care was provided for patients as directed by the primary pulmonologist. Study medication and placebo were administered using a Pari LC Plus ${ }^{\mathrm{TM}}$ jet nebulizer and a Pulmo-Aide ${ }^{\mathbb{R}}$ compressor. Both TSI and placebo were supplied by Novartis. The University of Michigan investigational drug services performed the randomization and double blinding for the study.

HRCT and spirometry (Sensormedics, $\mathrm{V}_{\max }$ 6200, Yorba Linda, CA), were obtained at baseline, $28 \pm 2$ days and at the end of the study \pm 4 days. Spirometry was obtained according to ATS guidelines. CXR was done at baseline for evaluation of lung disease and as part of routine annual care. Patients were enrolled at the time of their annual visit. Throat/sputum cultures were obtained prior to enrollment for confirmation of colonization with P. aeruginosa. A CF quality of life questionnaire (CFQ-R), validated for use in $\mathrm{CF}$ patients, ${ }^{15}$ was administered at baseline and at the end of the study (visit 3 ) to patients and parents/guardians. Adverse events, hospitalizations, concomitant medication use, and school/work days missed due to illness, were recorded during the study.

HRCT scan was performed using $40 \mathrm{~mA}, 1 \mathrm{sec}$ gantry rotation time, and $120 \mathrm{kVp}$ for each slice through the lung. Utilizing a HiSpeed Advantage ${ }^{\mathbb{R}}$ scanner (General Electric Medical Systems, Milwaukee, WI), images were obtained at $1 \mathrm{~mm}$ thickness. Five equally spaced images in inspiration and in expiration, after an AP inspiratory scout view, were obtained for a total of 10 images. Patients were placed in the supine position and instructed to take a deep breath and hold it for 7-10 sec. During each breath hold, the five inspiratory sections were obtained. The same technique was repeated after patients were asked to exhale to lowest volume and hold their breath for the expiratory sections. Patients practiced the techniques prior to the testing and were monitored by both the radiology technician and the research coordinator. If techniques were suboptimal, patients were instructed to repeat them. The superior-most image was at the upper margin of the posterior 3rd rib and the inferior-most image was $1 \mathrm{~cm}$ above the diaphragm. The range of the effective radiation dose was estimated to be $0.03-0.05 \mathrm{mSv}$ and the equivalent radiation dose to the entire lung was estimated to be $0.09 \mathrm{mGy}$ for each HRCT study. HRCT images were reviewed on a workstation (Sienet Magicview, version 42, Siemens, New York, NY). Images were initially displayed with a window width of 1,000 Hounsfield units (HU) and level of $-600 \mathrm{HU}$; however, the readers were permitted to modify the window and level of display of individual images and examinations for optimal viewing.

Scoring of the HRCT scans and CXRs were performed by two pediatric radiologists (PJS and BPE). Both readers were blinded to patient name, number, and study date at the time of reading and scoring. Each CXR (conventional analog) was scored twice by each radiologist using the Brasfield scoring method, ${ }^{16}$ and the scores were averaged for the two readers. A CXR score of 25 points indicates a normal finding, the score decreasing with increasing severity of lung disease. The HRCT score was derived from Brody and others. ${ }^{6,17-20}$ The score was then modified by dropping two component categories; atelectasis and inhomogeneity as discussed in our previous work. $^{14}$ Each reader read each HRCT twice in a randomized, blinded fashion. Means of the scores for each reader were calculated separately and compared between baseline and 28 days and between baseline and end of study. Scores of both readers were also combined (four total readings); a mean of the combined score was calculated and compared between baseline and 28 days and between baseline and end of study. One reader read the HRCT scans for the second time within 12 weeks from the first reading. The second reader read the HRCT scans for the second time 1-2 months after the first reading.

Each lung (right and left) was scored for each of the five imaging levels. Six imaging component categories were scored at each level for each lung: nodular opacities, linear opacities, hyperinflation (lack of normal decrease in volume on expiratory views), peribronchial thickening (abnormal thickening of bronchial walls), bronchiectasis (dilated bronchi usually judged relative to diameter of adjacent pulmonary artery branch), and mucus plugging (opacified bronchus/bronchi). Hyperinflation was scored on expiration since the expiratory images showed this finding better. The other five component categories were scored on inspiratory images. Scoring ranged from 0 to 4 with 0 indicating a normal finding and 4 indicating severe disease. The HRCT final score of each patient was calculated based on the severity and extent of the morphologic changes, and represented the score from the two lungs and was averaged for two readers. Three summary scores were calculated. (1) A component category score: the average of both readers' scores across the five levels within each category and for both lungs. (2) An imaging level score: The average of both readers' scores across six components within each level and for both lungs. (3) Total score: the sum of all six total HRCT category scores or the sum of all five level scores. Each subscore (i.e., component category score) could range from 0 to 80 [score of $80=$ score of each of the five levels for each lung (10 scores) which varied from 0 to 4 (maximum of 40$) \times 2$ lungs]. The score was the average of the four readings for the two readers. Potential total scores varied from 0 to 480 .

\section{Statistical Analysis}

The primary aim of this study was to evaluate response to TSI (vs. placebo) using both HRCT and PFTs in order to obtain preliminary data regarding the relative value of HRCT as an objective measure of treatment response. 
While the previous publication from this project evaluated this related to initial treatment response (i.e., immediately following first TSI treatment) ${ }^{14}$ this manuscript describes the results pertaining to longer-term treatment response (i.e., approximately 30-40 days after completion of third TSI treatment).

Initial data analysis was performed using descriptive statistics. Frequencies and percentages were calculated for categorical variables; mean, median, standard deviation, minimum, and maximum were calculated for continuous variables. HRCT data were analyzed as both absolute score (for individual components and composite) and as percent of maximum score. HRCT data are presented herein as percent of maximum score throughout. As this is a pilot study, power calculations were not performed. The number of subjects studied was arbitrarily chosen. The baseline analysis was performed with 16 patients in each treatment group. End of study analyses included data from 16 patients in the TSI arm and 15 patients in the placebo arm (excluding the drop-out subject in the placebo arm). SAS version 8 software was utilized for all analyses. Between-group comparisons were performed using both the Student's $t$-test and the nonparametric Wilcoxon rank-sum test for continuous data and using the Chi-squared test for categorical data. Within-individual comparisons of continuous data (e.g., visits 2 and 3 vs. visit 1 within each treatment group) were performed using the paired $t$-test. Pearson's correlation coefficient was used to describe the relationship between continuous quality-of-life data and other continuous data. All applicable statistical tests used were two-sided, and $P$ values $\leq 0.05$ were considered statistically significant. Coefficient of variation and test of differences in mean scores between and within readers (mean $\pm \mathrm{SD}$ ) were used to measure inter/intra-reader variability in scoring CXR and HRCT images. Inter/intra-reader reliability analyses were carried out to test whether there was a difference between the reliability of the scoring for HRCT and CXR. The subjects in both groups were combined to enlarge the sample size for statistical analyses. HRCT scores from three visits and CXR scores from the first visit were averaged for these analyses. Analysis was also done for the total HRCT score, all HRCT subscores, and the CXR scores.

The HRCT total score, $\mathrm{FEF}_{25-75 \%}$ predicted and $\mathrm{FEV}_{1} \%$ predicted changes during the study were analyzed using a mixed model analysis. The analysis was done where time was in the unit of days with time $=0$ for baseline, time $=28-31$ for visit 2 , and time $=168-176$ for visit 3 . Treatment $=0$ represented placebo and Treatment $=1$ represented TSI. The analysis evaluated the change in mean total HRCT score, $\mathrm{FEF}_{25-75 \%}$, and $\mathrm{FEV}_{1} \%$ predicted for both groups with 1 day increase in time for the duration of the study. This analysis was done to evaluate the cumulative effect of using TSI versus placebo in this patient population over the duration of the study.

A frequency distribution of HRCT score differences was also conducted for the individual scores, by patient and by case over the three visits to demonstrate the pattern of scoring for each of the two readers. Ninety-seven percent of the observations had a maximum score difference of 1 or less. Maximum difference $=($ maximum of the four scores, E1, E2, S1, S2) - (minimum of the four scores E1, E2, S1, S2).

\section{RESULTS}

Thirty-two patients were enrolled and randomized to one of the two treatment groups. Thirty-one patients completed the study. One patient in the placebo group dropped out of the study after few days of enrolling due to bad taste of the "medication." The median overall treatment adherence rate was $97.6 \%$, with only three subjects having adherence rates of less than $87.5 \%$ (one in TSI group with a $43.5 \%$ adherence rate, and two in placebo group with $59.5 \%$ and $21.4 \%$ adherence rates).

Baseline clinical characteristics of the two groups are presented in Table 1. Most of the study population had mild to moderate lung disease as indicated by the mean CXR Brasfield score, mean percent predicted $\mathrm{FEV}_{1}$, and $\mathrm{FEF}_{25-75 \%}, 21.55,89.7 \%$, and $75.9 \%$, respectively. CXR was done only at baseline to evaluate disease severity and as part of routine CF care. Patients randomized to TSI were younger and had milder lung disease than those randomized to Placebo. However, there were no significant differences between the two groups except for weight.

The intra-observer difference in the mean HRCT score for the first reader was $P=0.76$ and for the second reader the $P$-value was 0.48 . Non-parametric tests (Wilcoxon rank-sum) values were 0.51 and 1.00 , respectively, which were consistent with the $t$-test results. When both readers' scores were combined, $t$-test and Wilcoxon rank-sum $P$ values were 0.80 and 0.64 , respectively. The interval between the two readings for the first reader was 12 weeks while the interval for the second reader was 12 months.

Mean $( \pm$ SD) time between end of first TSI treatment and evaluation was $1.4( \pm 2.0)$ days in the TSI group and $1.4( \pm 1.5)$ days in the placebo group. Mean time between the end of the third TSI treatment and evaluation was 30.2 $( \pm 8.0)$ days in the TSI group and $29.5( \pm 8.2)$ days in the placebo group. The total HRCT and six component scores for each visit and the difference between visits 1 and 2 and visits 1 and 3 are illustrated in Tables 2A and 2B. Using two tailed paired $t$-test, the percent maximum HRCT score decreased by $1.4 \pm 2.6 \%$ (mean $\pm \mathrm{SD})(P=0.049)$ and $0.3 \pm 2.8 \%(P=0.63)$ for the TSI group and decreased by $0.1 \pm 1.5 \%,(P=0.74)$ and increased by $0.6 \pm 1.8 \%$, 
TABLE 1-Baseline Characteristics of Study Population

\begin{tabular}{lcc}
\hline & TSI & Placebo \\
\hline Number of patients & 16 & 16 \\
Gender (M/F) & $6 / 10$ & $6 / 10$ \\
Age (years) & $11.81 \pm 7.46^{2}(6.0-34.7)^{3}$ & $15.86 \pm 7.25(7.4-28.8)$ \\
Weight (kg) & $34.83 \pm 14.08(17.6-62.0)$ & $46.38 \pm 16.92(21.9-73.3)$ \\
Weight for age (percentile) & $25 \%$ & $10 \%$ \\
FEV 1 predicted & $95.73 \pm 17.21(55.0-134.1)$ & $83.71 \pm 21.07(40.00-108.73)$ \\
FEF $25 \%-75 \%$ predicted & $81.71 \pm 22.94(29.35-111.83)$ & $70.04 \pm 31.19(11.40-110.14)$ \\
Total HRCT score & $23.47 \pm 14.60(6.75-60.5)$ & $25.10 \pm 17.81(5.00-73.25)$ \\
Total HRCT score $\%$ of maximum score & $4.89 \pm 3.04 \%(1.41-12.60 \%)$ & $5.23 \pm 3.71 \%(1.04-15.06)$ \\
CXR Brasfield score & $22.05 \pm 1.32(18.8-24)$ & $21.05 \pm 2.88(14.8-25)$ \\
Sweat chloride (meg/L) & $101.1 \pm 17.75(70.0-134.0)$ & 0.24 \\
Genetic analysis & 0.78 & 0.78 \\
$\quad$ Homozygous delta F508 & 8 & 0.22 \\
$\quad$ Heterozygous delta F508 & 2 & 0.58 \\
\hline
\end{tabular}

${ }^{1}$ Student's $t$-test for two independent samples (two tailed).

${ }^{2}$ Mean $\pm \mathrm{SD}$.

${ }^{3}$ Range.

$(P=0.23)$ for the placebo group between visits 1 and 2, and visits 1 and 3 , respectively. Figure $1 \mathrm{~A}$ and $\mathrm{B}$ represents Total HRCT score for individual patients in TSI and placebo groups, respectively. Most subjects had mild lung disease as indicated by the low total HRCT score. Patients with more severe disease showed significant improvement in total HRCT score $(\mathrm{n}=1)$ and no change in total HRCT score in the placebo group $(\mathrm{n}=2)$.

The mean difference between visits 2 and 1 and visits 3 and 1 for $\mathrm{FEF}_{25-75 \%}$ for the TSI groups were $4.54 \pm 21.60$ $( \pm \mathrm{SD})(P=0.23)$ and $-1.79 \pm 26.27(P=0.64)$ and for placebo group were $-0.40 \pm 8.33(P=0.41)$ and $3.87 \pm 22.88(P=0.58)$. The mean difference between visits 2 and 1 and visits 3 and 1 for $\mathrm{FEV}_{1}$ for the TSI group were $0.60 \pm 14.15(P=0.70)$ and $-4.13 \pm 17.75$ $(P=0.43)$ and for placebo group were $1.33 \pm 6.18$ $(P=0.48)$ and $2.20 \pm 8.50(P=0.24)$. Figure 2 illustrates some of the inspiratory images of one patient that was randomized to TSI. Patient had moderate lung disease with $\mathrm{FEV}_{1}$ of $70 \%$ of predicted.

The mixed model analysis results are summarized in Table 3 . The $P$-value for the TSI group between visits 1 and 2 was statistically significant $(P=0.02)$ in this model. There was some improvement (not statistically significant) in the HRCT score for the TSI group between visits 1 and $3(P=0.22)$ and in the $\mathrm{FEF}_{25-75 \%}$ for the TSI group between visits 1 and $2(P=0.12)$.

There was no statistical difference between the two readings of HRCT for each of the two readers (intraobserver difference) using either parametric (Student's $t$-test) or non-parametric (Wilcoxon rank-sum) statistical tests. In addition, there was no statistical difference between the two readers values (inter-observer variability).

Table 4 summarizes the antibiotics use during the study period. The antibiotics use was a marker for pulmonary exacerbation during the study period. There was statistical significance between the TSI and placebo groups for the total antibiotic courses, oral antibiotics courses, and days on oral antibiotics with $P=0.012,<0.01$, and $<0.01$, respectively. CFQ-R data were analyzed. There was a poor correlation $(r=0.28)$ between CFQ-R and HRCT score. In addition, there was no statistical significance between baseline and end of study for both groups.

\section{DISCUSSION}

In this pilot study, there was significant improvement in HRCT score after 28 days of TSI versus no improvement in placebo group. At the end of the study (approximately 28-36 days after completion of study medication), however, there was no significant change in HRCT score. This lack of improvement could be due to the severalweek delay between completion of the study medication and the final study examination. Alternatively, this could indicate that the most significant improvement in HRCT score in this mostly mild patient population occurred after the first cycle of TSI. It has been reported that TSI is most effective in patient with moderate to severe $\left(\mathrm{FEV}_{1} 25-\right.$ $75 \%$ predicted) lung disease. ${ }^{12}$ This is the first study, to the best of our knowledge, that used HRCT to evaluate the short-term (28 days) and long-term effects (after three cycles) of TSI in mild CF patients.

The limitation of only five cuts for inspiratory and five expiratory HRCT scans may have been another cause of limiting the ability to discern significant difference after the three cycles of TSI treatment in this mild patient population. The use of limited cuts reduces the sensitivity to detect changes as documented recently. ${ }^{9,21-23}$ However, using limited cuts reduces radiation dosing that patients are exposed to. In this protocol, there were specific instructions on how and where to place the five 

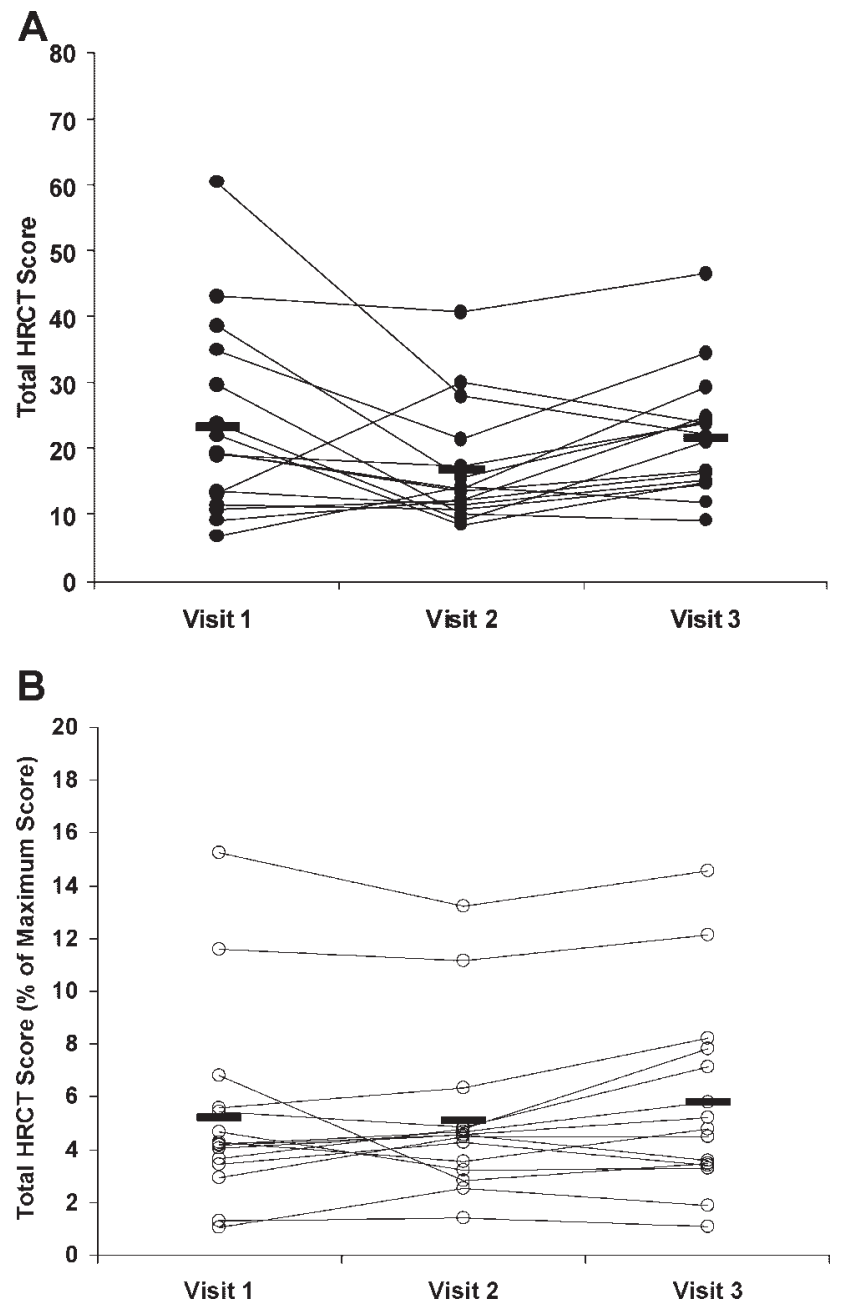

Fig. 1. A: Total HRCT score for individual subjects in the TSI group. B: Total HRCT score for individual subjects in the placebo group. Solid symbols, TSI group; open symbols, placebo; solid bars, mean values for each visit.

slices at each study. This should result on each slice being in the same vicinity of the previous one, but not the exact position. The choice of the limited number of cuts to five scans during inspiration and five during expiration was determined following our previous work. ${ }^{5}$ The number of cuts in this study was thought to be reasonably representative of the disease present and changes that occurred. The effective and equivalent doses of radiation from HRCT in this study was approximately equal to two CXRs (PA and lateral view each), even though the radiation dose is delivered in a different manner for both procedures (effective dose was 0.04 and $0.02 \mathrm{mSv}$ and the equivalent doses were 0.09 and $0.04 \mathrm{mGy}$, respectively). Axial HRCT images in inspiration at two levels from a patient in the TSI group at baseline, 28 days and at end of study are shown in Figure 2. The changes in HRCT score by each reader and by both reader were analyzed. The intra-observer variability was higher with the longer interval (1-2 months) between the two readings versus
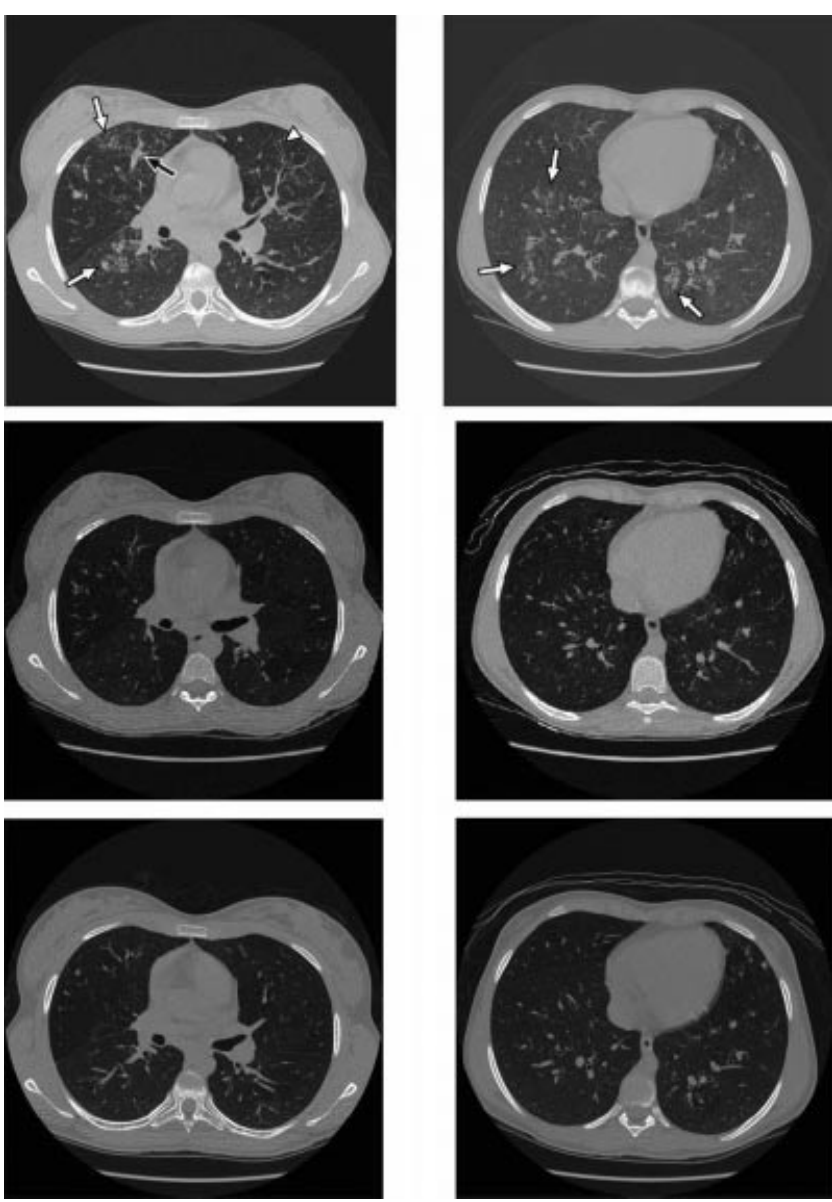

Fig. 2. A-F: Inspiratory HRCT images obtained at baseline $(A, B)$, at 28 days (C,D), and at the end of study (E,F). Images A, C, and $E$ correspond as the middle of five images (Level 3 ). Images $B, D$, and $F$ correspond as the lowest of five images (Level 5). The initial images $(A, B)$ show nodular opacities (white arrow), bronchiectasis (arrowhead), mild bronchial wall thickening and mucous plugging (black arrow). Findings are dramatically improved at 28 days $(C, D)$ and demonstrate slight additional improvement at the end of study $(E, F)$.

lower variability with the shorter interval (1-2 weeks). This is consistent with prior observation. ${ }^{24}$

Changes in HRCT component scores for individual patients are shown in Figure 1A and B. Changes of the total HRCT score and component scores are summarized in Tables 2A and 2B. In this study, it was noted that peribronchial thickening, hyperinflation, and linear opacities were the most useful component scores, overall. Nodular opacities and bronchiectasis seemed only marginally useful, and mucus plugging was of little value. For comparing treated versus non-treated patients (i.e., TSI vs. placebo), peribronchial thickening seemed best (only component to show significant differences, both early (V2) and later (V3) after treatment), with hyperinflation (measure showing biggest absolute difference) and linear opacities (relatively large differences and one of least 
TABLE 3-Mixed Model: Mean Changes in HRCT Score, FEF $_{25-75 \%}$ and FEV $_{1} \%$ Predicted With 1 Day Increase in Time

\begin{tabular}{|c|c|c|c|c|}
\hline & \multicolumn{2}{|c|}{ TSI } & \multicolumn{2}{|c|}{ Placebo } \\
\hline & Mean change & $P$-value & Mean change & $P$-value \\
\hline \multicolumn{5}{|c|}{ HRCT score } \\
\hline $\mathrm{V} 1-\mathrm{V} 2$ & -0.24 & 0.02 & -0.01 & 0.93 \\
\hline $\mathrm{V} 1-\mathrm{V} 3$ & -0.03 & 0.22 & 0.02 & 0.29 \\
\hline \multicolumn{5}{|c|}{$\mathrm{FEF}_{25-75 \%}$ predicted } \\
\hline $\mathrm{V} 1-\mathrm{V} 2$ & 0.29 & 0.12 & -0.05 & 0.69 \\
\hline $\mathrm{V} 1-\mathrm{V} 3$ & -0.02 & 0.72 & 0.01 & 0.76 \\
\hline \multicolumn{5}{|c|}{$\mathrm{FEV}_{1} \%$ predicted } \\
\hline $\mathrm{V} 1-\mathrm{V} 2$ & 0.08 & 0.50 & -0.01 & 0.96 \\
\hline $\mathrm{V} 1-\mathrm{V} 3$ & -0.01 & 0.61 & 0.00 & 0.83 \\
\hline
\end{tabular}

V, visit.

variable measures) also of some value. For evaluating treatment response within an individual patient, linear opacities seemed best (only significant difference in paired analysis of TSI group), with hyperinflation (biggest absolute difference) and peribronchial thickening (large difference and relatively low variability) also useful. Of interest, the baseline bronchiectasis score was third highest (following peribronchial thickening and hyperinflation) at $9.9 \pm 6.2 \%$ and $13.4 \pm 10.3 \%$ for TSI and placebo group, respectively. That indicates that in this mostly mild patient population, the changes in the lungs as indicated by HRCT are more severs than expected.

There was significant improvement in HRCT score after 28 days of TSI treatment, while there was no significant improvement using $\mathrm{FEF}_{25-75 \%}$ or $\mathrm{FEV}_{1} \%$ predicted. Using HRCT as an objective measure to evaluate treatment effect in $\mathrm{CF}$ patients with mild lung disease could be a reasonable alternative to pulmonary function testing, first in the research setting and eventually in the clinical setting once the scoring system and technique are standardized. These results were not surprising since pulmonary function testing provides a global measure of lung function, whereas, on the other hand, areas with localized abnormalities can be feasibly demonstrated on HRCT scans. ${ }^{25}$
It has been reported that HRCT is a more sensitive measure in detecting early lung disease in $\mathrm{CF}^{8-11}$ It is also reported that in pediatric bronchiectasis of different etiologies, substantial structural damage was evident on HRCT scores in the absence of abnormalities in pulmonary function testing. ${ }^{26-28}$ HRCT has been used as an end point for therapeutic trials. In a previous study we evaluated the efficacy of rhDNase in $\mathrm{CF}$ patients younger than 5 years of age using HRCT and CXR as end points. ${ }^{5}$ We concluded that administration of rhDNase was associated with improvement in the HRCT in this young CF population compared to placebo group while CXR score was not significantly different between the two groups. ${ }^{5}$ In another study, rhDNase intervention versus placebo in patients with mild CF lung disease was done. ${ }^{29}$ A spirometer-triggered HRCT and spirometry were used as end points. The conclusion from this study was that quantitative air trapping is a more consistent sensitive outcome measure than either spirometry or total HRCT scores. It was able to discriminate differences in treatment effects in children with minimal CF lung disease. ${ }^{29}$

HRCT has been used as an outcome surrogate in few studies to evaluate acute pulmonary exacerbation and response to treatment. ${ }^{17,19,20}$ Mucus plugging, centrilobular nodules, peribronchial thickening, and overall

TABLE 4-Antibiotics Use During the Study Period

\begin{tabular}{lccc}
\hline & TSI & Placebo & $P$-value \\
\hline Number & 16 & 15 & - \\
Total antibiotic courses & $22(1.38 \pm 1.86)$ & $42(2.8 \pm 1.42)$ & 0.012 \\
Oral antibiotic courses & $21(1.31 \pm 1.78)$ & $41(2.73 \pm 1.39)$ & $<0.01$ \\
Days on oral antibiotics & $277(17.3 \pm 25.4)$ & $721(48.1 \pm 45.8)$ & $<0.01$ \\
Intravenous antibiotic courses & 1 & 1 & $\mathrm{NS}$ \\
Number of hospitalizations & 1 & 1 & $\mathrm{NS}$ \\
Days on intravenous antibiotics & 21 & 24 & $\mathrm{NS}$ \\
\hline
\end{tabular}

$\mathrm{NS}=$ not significant $(>0.05)$. 
appearance were potentially reversible findings in symptomatic patients in all three studies. ${ }^{17,19,20}$ HRCT accurately revealed disease severity and correlated with clinical improvement as determined by $\mathrm{PFT}^{20}$

Oral antibiotic courses and days on antibiotics were both significantly less in the TSI versus placebo group $(P<0.01)$ (Table 4). There was one course of intravenous antibiotics and one hospitalization in each group. The significant difference in the oral antibiotic use and days on oral antibiotics with no difference on intravenous antibiotics could be due to the mostly mild patient population. Pulmonary exacerbations in the study subjects were mild in nature and were successfully treated with oral antibiotics. That is in contrast to the moderate-severe patient population which showed significant difference between the two groups, in the use of intravenous antibiotics and hospitalization rate which indicated more severe pulmonary exacerbations. ${ }^{12}$ This finding could be justification for future study to evaluate the usefulness of different antibiotic treatment modalities in this mild CF patient population.

Analysis of objective (HRCT score) and subjective (CFQ-R) outcomes was done between baseline and end of study. Analysis was stratified by baseline HRCT score. There was an overall poor correlation $(r=0.28)$ between CFQ-R and HRCT score. Removal of outliers from the analysis only modestly improved the correlation (increase $\mathrm{r}$ from 0.28 to 0.34 ). Best correlation between change in HRCT and change in CFQ-R was for those in the middle percentile of baseline HRCT score (i.e., 20.25-32.35) with correlation of $r=0.58-0.8$. An evaluation of parents/ care givers-assessed CFQ-R for patients $6-13$ years of age versus patients-assessed CFQ- $\mathrm{R}$ was done. There was a general agreement between the two groups $(r=0.62)$. This close relationship is identical in TSI and placebo groups. Explanations of lack of improvement in CFQ-R could be due to the small number of patients in this pilot study. In addition, it could have been influenced by the time spent performing the treatment and the unpleasant taste of medication. One could conclude that using subjective measures like CFQ-R to detect TSI treatment effect has to be used with caution. The objective measures (HRCT score, $\mathrm{FEF}_{25-75 \%}$ and $\mathrm{FEV}_{1}$ ) all correlated well with one another, but poorly with the subjective measures (CFQ-R).

Limitations of the study include:

1. This is a pilot study with small sample size in subject groups. A larger study would be of interest to further evaluate the usefulness of HRCT in this patient population. Another complicating factor in the interpretation of these findings is that patients randomized to TSI were generally younger and had milder lung disease (though not to a statistically significant degree).
2. The use of different time intervals between the end of a treatment and examination (i.e., approximately 1.4 days for V2 vs. 30 days for V3) complicates any attempted direct comparison of first-cycle versus third-cycle response.

3. Findings on expiratory images (hyperinflation) were dependent on the patient following breath holding instructions closely. A suboptimal expiratory effort could mimic hyperinflation. The importance of monitoring volume has been reported. ${ }^{19,29}$ Lack of adequate volume monitoring may have increased the variability of this potentially useful component.

4. The use of limited HRCT cuts could have been a reason that led to reduction of the sensitivity to detect changes at the end of the study. In addition, that could have limited the matching of airways and regional parenchyma on serial HRCT scans during the study duration.

\section{CONCLUSION}

- HRCT can be a sensitive measure of change in mild CF pulmonary disease.

- HRCT is likely to require a smaller sample size than $\mathrm{FEV}_{1}$ or $\mathrm{FEF}_{25-75 \%}$ to demonstrate a significant treatment effect in patients with mild lung disease especially after first 28 days on TSI.

- The limited number of slices with low $\mathrm{mA}$ and/or scan time reduces the radiation dose.

- CFQ-R may not be a useful measure in detecting a TSI treatment difference in this mostly mild patient population.

- The use of antibiotics, especially oral and number of days on antibiotics is a useful end point in evaluating treatment effect in this patient population.

\section{ACKNOWLEDGMENTS}

We would like to thank Bruce Brock, PhD, MPH and Jinyao Zhang for their help with the statistical analysis and Cindy Benedict and Sarah Chaudhry for administrative assistance.

\section{REFERENCES}

1. Brody AS. Early morphologic changes in the lungs of asymptomatic infants and young children with cystic fibrosis. J Pediatr 2004;144:145-146.

2. Gibson RL, Burns JL, Ramsey BW. Pathophysiology and management of pulmonary infections in cystic fibrosis. Am J Respir Crit Care Med 2003;168:918-951.

3. Cystic Fibrosis Foundation. Patient registry, 2007 annual data report. Bethesda, MD: Cystic Fibrosis Foundation; 2008.

4. Davis SD, Fordham LA, Brody AS, Noah TL, Retsch-Bogart GZ, Qaqish BF, Yankaskas BC, Johnson RC, Leigh MW. Computed tomography reflects lower airway inflammation and tracks 
changes in early cystic fibrosis. Am J Respir Crit Care Med 2007; 175:943-950.

5. Nasr SZ, Kuhns LR, Brown RW, Hurwitz ME, Sanders GM, Strouse PJ. Use of computerized tomography and chest X-rays in evaluating efficacy of aerosolized recombinant human DNase in cystic fibrosis patients younger than age 5 years: a preliminary study. Pediatr Pulmonol 2001;31:377-382.

6. Santamaria F, Grillo G, Guidi G, Rotondo A, Raia V, de Ritis G, Sarnelli P, Caterino M, Greco L. Cystic fibrosis: when should high-resolution computed tomography of the chest be obtained? Pediatrics 1998;101:908-913.

7. Tiddens HAWM. Chest computed tomography scan should be considered as a routine investigation in cystic fibrosis. Paediatr Respir Rev 2006;7:202-208.

8. De Jong PA, Nakano Y, Lequin MH, Mayo JR, Woods R, Paré PD, Tiddens HAWM. Progressive damage on high resolution computed tomography despite stable lung function in cystic fibrosis. Eur Repir J 2004;23:93-97.

9. De Jong PA, Nakano Y, Hop WC, Long FR, Coxson HO, Paré PD, Tiddens HAWM. Changes in airway dimensions on computed tomography scan in children with cystic fibrosis. Am J Respir Crit Care Med 2005;172:218-224.

10. Marchant JM, Masel JP, Dickinson FL, Masters IB, Chang AB. Application of chest high-resolution computed tomography in young children with cystic fibrosis. Pediatr Pulmonol 2001;31: 24-29.

11. Brody AS, Klein JS, Molina PL, Quan J, Bean JA, Wilmott RW. High-resolution computed tomography in young patients with cystic fibrosis: distribution of abnormalities and correlation with pulmonary function tests. J Pediatr 2004;145:32-38.

12. Ramsey BW, Pepe MS, Quan JM, Otto KL, Montgomery AB, Williams-Warren J, Vasiljev K-M, Borowitz D, Bowman CM, Marshall BC, Marshall S, Smith AL. Intermittent administration of inhaled tobramycin in patient with cystic fibrosis. N Engl J Med 1999;340:23-30.

13. Murphy TD, Anbar RD, Lester LA, Nasr SZ, Nickerson B, VanDevanter DR, Colin AA. Treatment with tobramycin solution for inhalation reduces hospitalizations in young CF subjects with mild lung disease. Pediatr Pulmonol 2004;38:314-320.

14. Nasr SZ, Gordon D, Sakmar E, Yu X, Christodoulou E, Eckhardt BP, Strouse PJ. High resolution computerized tomography of the chest and pulmonary function testing in evaluating the effect of tobramycin solution for inhalation in cystic fibrosis patients. Pediatr Pulmonol 2006;41:1129-1137.

15. Quittner AL, Sweeny S, Watrous M, Munzenberger P, Bearss K, Nitza AG, Fisher LA, Henry B. Translation and linguistic validation of a disease-specific quality of life measure for cystic fibrosis. J Pediatr Psychol 2000;25:403-414.

16. Brasfield D, Hicks G, Seng-Jaw S, Tiller RE. The roentgenogram in cystic fibrosis: a new scoring system. Pediatrics 1979;63:24-29.
17. Brody AS, Molina PL, Klein JS, Rothman BS, Ramagopal M, Swartz DR. High-resolution computed tomography of the chest in children with cystic fibrosis: support for use as an outcome surrogate. Pediatr Radiol 1999;29:731-735.

18. Robinson TE, Leung AN, Northway WH, Blankenberg FG, Chan FP, Bloch DA, Holmes TH, Moss RB. Composite spirometric-computed tomography outcome measure in early cystic fibrosis lung disease. Am J Respir Crit Care Med 2003; 168:588-593.

19. Robinson TE, Leung AN, Northway WH, Blankenberg FG, Bloch DA, Oehlert JW, Al-Dabbagh H, Hubli S, Moss RB. Spirometrytriggered high-resolution computed tomography and pulmonary function measurements during an acute exacerbation in patients with cystic fibrosis. J Pediatr 2001;138:553-559.

20. Shah RM, Sexauer W, Ostrum BJ, Fiel SB, Friedman AC. Highresolution $\mathrm{CT}$ in the acute exacerbation of cystic fibrosis: evaluation of acute findings, reversibility of those findings, and clinical correlation. AJR 1997;169:375-380.

21. de Jong PA, Nakano Y, Lequin MH, Tiddent HAWM. Dose reduction for $\mathrm{CT}$ in children with cystic fibrosis: is it feasible to reduce the number of images per scan? Pediatr Radiol 2006;36:50-53.

22. Bankier AA, Mehrain S, Kienzl D, Weber M, Estene M, Gevenois PA. Regional heterogeneity of air trapping at expiratory thinsection CT of patients with bronchiolitis: potential implications for dose reductions and CT protocol planning. Radiology 2008; 247:862-870.

23. Goris ML, Robinson TE. Sampling density for the quantitative evaluation of air trapping. Pediatr Radiol 2009;39:221-225.

24. de Jong PA, Ottick MD, Robben SG, Lequin MH, Hop WCJ, Hendricks JJE, Paré PD, Tiddens HAWM. Pulmonary disease assessment in cystic fibrosis; comparison of ct scoring systems and value of bronchial and arterial dimension measurements. Radiology 2004;231:434-439.

25. Long FR, Williams RS, Castile RG. Structural airway abnormalities in infants and young children with cystic fibrosis. J Pediatr 2004; 144:154-161.

26. Santamaria F, Montella S, Camera L, Palumbo C, Greco L, Boner AL. Lung structure abnormalities, but normal lung function in pediatric bronchiectasis. Chest 2006;130:480-486.

27. Chang AB, Masel JP, Boyce NC, Wheaton G, Torzillo PJ. Non$\mathrm{CF}$ bronchiectasis: clinical and HRCT evaluation. Pediatr Pulmonol 2003;35:477-483.

28. Pifferi M, Caramella D, Bulleri A, Baldi S, Peroni D, Pietrobelli A, Boner AL. Pediatric bronchiectasis: correlation of HRCT, ventilation and perfusion scintigraphy, and pulmonary function testing. Pediatr Pulmonol 2004;38:298-303.

29. Robinson TE, Goris ML, Zhu HJ, Chen X, Bhise P, Sheikh F, Moss RB. Dornase alfa reduces air trapping in children with mild cystic fibrosis lung disease. Chest 2005;185:2327-2335. 